\title{
BIODIGESTÃO ANAERÓBIA DE DEJETOS DE SUÍNOS COM E SEM SEPARAÇÃO DA FRAÇÃO SÓLIDA EM DIFERENTES TEMPOS DE RETENÇÃO HIDRAÚLICA
}

\author{
MARCO A. P. ORRICO JÚNIOR ${ }^{1}$, ANA C. A. ORRICO ${ }^{2}$, JORGE DE LUCAS JÚNIOR ${ }^{3}$
}

RESUMO: O objetivo deste trabalho foi avaliar o processo de biodigestão anaeróbia utilizando dejetos de suínos, com e sem separação da fração sólida, considerando-se diferentes tempos de retenção hidráulica. Para tanto, foram utilizados biodigestores tubulares semicontínuos abastecidos com água residuária de suinocultura, com e sem separação da fração sólida (CSFS e SSFS, respectivamente), manejados com tempos de retenção hidráulica (TRH) iguais a 36; 29; 22 e 15 dias. A eficiência dos tratamentos foi avaliada pela redução dos números mais prováveis de coliformes totais e fecais, teores de fibra em detergentes neutro e ácido, demandas química e bioquímica de oxigênio e dos potenciais de produção de biogás e metano. A qualidade do biofertilizante foi avaliada quanto aos teores de macro e micronutrientes. A separação da fração sólida acarretou decréscimo nos teores de fibra dos afluentes, o que contribuiu para o aumento da eficiência da produção de metano. Foram observados valores de 0,47 e $0,75 \mathrm{~m}^{3} \mathrm{CH}_{4} \mathrm{~kg}^{-1} \mathrm{SV}$ adicionado para os afluentes SSFS e CSFS, respectivamente, no TRH de 15 dias. Com o aumento do TRH, houve acréscimo médio de $50 \%$ no potencial de produção de metano $\mathrm{kg}^{-1}$ de SV adicionado. Não foram observadas diferenças significativas nas reduções de coliformes fecais e totais, sendo a maior redução de 3,6 $10^{9}$ para 3,6 $10^{2} \mathrm{NMP} 100 \mathrm{~mL}^{-1}$ para o TRH de 36 dias CSFS.

PALAVRAS-CHAVE: coliformes, sólidos totais, TRH.

\section{ANAEROBIC BIODIGESTION OF SWINE MANURE WITH AND WITHOUT SEPARATION OF THE SOLID FRACTION IN DIFFERENT HYDRAULIC RETENTION TIMES}

\begin{abstract}
This work aimed to evaluate the anaerobic biodigestion process by using swine manure with and without separation of the solid fraction and considering different hydraulic retention times. For such purpose semi continual plug flow biodigestors were used, with residual water from a pig farm with and without separation of the solid fraction, managed with 36; 29; $22 \mathrm{e}$ 15 days of hydraulic retention. Efficiency of the treatments was evaluated by the reduction of the most probable number of total and fecal coliforms, fiber content, chemical and biochemical oxygen demands, besides the biogas and methane production potential and biofertilizer quality. The separation of the solid fraction decreased in fiber content from effluents, what contributed to an increase in the efficiency in the methane production. The observed values were: 0.47 and $0.75 \mathrm{~m}^{3}$ $\mathrm{CH}_{4} \mathrm{~kg}^{-1} \mathrm{VS}$ added to the effluent with and without separation of the solid fraction respectively, in the HRT of 15 days. With the increase of HRT there was a medium increase of $50 \%$ in the production of potential of methane $\mathrm{kg}^{-1} \mathrm{VS}$ added. It was not observed significant differences in reduction of fecal and total coliforms. The largest reduction was $3.610^{9}$ to $3.610^{2} \mathrm{NMP} 100 \mathrm{~mL}^{-1}$ for the 36 days HRT.
\end{abstract}

KEYWORDS: coliforms, total solids, HRT, swine manure.

\footnotetext{
${ }^{1}$ Zootecnista, Doutorando em Zootecnia, Faculdade de Ciências Agrárias e Veterinárias - UNESP, Jaboticabal - SP, marcoorrico@yahoo.com.br

${ }^{2}$ Zootecnista. Profa. Adjunta, Faculdade de Ciências Agrárias - UFGD, Dourados - MS, anacarolamorim@ hotmail.com

${ }^{3}$ Eng $^{\mathrm{o}} \mathrm{Agr}^{\mathrm{o}}$, Prof. Titular, Faculdade de Ciências Agrárias e Veterinárias - UNESP, Jaboticabal - SP, jlucas @ fcav.unesp.br Recebido pelo Conselho Editorial em: 8-4-2008

Aprovado pelo Conselho Editorial em: 7-9-2009
} 


\section{INTRODUÇÃO}

Segundo dados da ABIPECS (2007), nos últimos cinco anos, houve aumento de $12 \%$ na produção mundial de carne suína, passando de 92 milhões de toneladas, em 2001, para aproximadamente 105 milhões, em 2006. A China lidera o mercado mundial, entre os maiores países produtores de carne suína, com 50 milhões de toneladas, ou seja, aproximadamente metade do total mundial, seguida pela União Europeia, que apresentou produção de 22 milhões de toneladas em 2006. Os Estados Unidos ocupam o terceiro lugar no ranking mundial com 9,6 milhões de toneladas, e na quarta posição está o Brasil, que fechou 2006 com produção de 3,2 milhões de toneladas de carne suína.

$\mathrm{Na}$ atualidade, muitos indicadores econômicos e produtivos da atividade agropecuária apontam para a maior concentração na produção de suínos no Brasil, sobretudo na região Sul. A concentração das unidades produtoras em uma mesma região (característica do sistema de integração) e a grande densidade de animais dentro das mesmas representam um desafio que tende a se agravar na suinocultura (AHNA et al., 2006). Os problemas ambientais relacionados com a atividade também se expressam de forma mais intensa em algumas regiões, porém a questão ambiental relacionada com o manejo de dejetos apresenta características que afetam toda e qualquer granja produtora.

Com base nas características quantitativas e qualitativas, torna-se evidente a necessidade de tratamento prévio dos resíduos produzidos por suínos para posterior aplicação no solo. A biodigestão anaeróbia pode ser utilizada nesse tratamento, pois, além de reduzir o poder poluente e os riscos sanitários dos dejetos, também gera como subprodutos o biogás e o biofertilizante (ALVAREZ \& GUNNAR, 2008). O processo de biodigestão anaeróbia consiste na otimização da degradação da matéria orgânica contida nos dejetos, permitindo, também, a redução das demandas química e bioquímica de oxigênio e de sólidos voláteis, tornando os nutrientes mais disponíveis para as plantas (CÔTÉ et al., 2006; ORRICO et al., 2007).

Apesar dos dejetos de suínos serem de alta biodegradação, uma fração desse total pode não ser biodegradável ou lentamente biodegradável. RICO et al. (2006) verificaram que a separação da fração biodegradável para o tratamento anaeróbio resulta em maior eficiência, maior produção de biogás $\mathrm{kg}^{-1}$ de sólido, menor tempo de retenção hidráulica e maior economia, pois necessita de reatores de menor volume para o mesmo número de animais. Existem várias formas de separar frações mais degradáveis das porções menos degradáveis.

Em se tratando de água residuária, uma das formas mais comuns é a separação das frações sólida e líquida, o que pode ser feito por meio de peneiras, tambores rotativos, centrifugação e decantação. A fração líquida possui maior quantidade de nutrientes solúveis e partículas de menor tamanho, ou seja, material mais facilmente degradável do que a fração sólida, que apresenta maior tamanho de partícula e frações menos degradáveis, como celulose, hemicelulose e lignina (RICO et al., 2006).

MOLLER et al. (2004) estudaram as formas de separação das frações sólidas dos efluentes de suinocultura e encontraram valores de biodegradabilidade de 30; 48 e 93\% para a fração sólida dos dejetos resultante da centrifugação, da precipitação química e da centrifugação, respectivamente.

Desta forma, o objetivo deste trabalho foi verificar o desempenho do processo de biodigestão anaeróbia, utilizando-se como substrato de água residuária de suinocultura, com e sem separação da fração sólida, e adotando-se diferentes tempos de retenção hidráulica (15; 22; 29 e 36 dias).

\section{MATERIAL E MÉTODOS}

A pesquisa foi desenvolvida no Laboratório de Biodigestão Anaeróbia do Departamento de Engenharia Rural, Faculdade de Ciências Agrárias e Veterinárias - FCAV/UNESP, Jaboticabal. Os dejetos foram produzidos por suínos em fase de crescimento e coletados do piso das baias por meio de raspagem. 
O preparo do substrato utilizado para o abastecimento dos biodigestores foi realizado no laboratório, misturando-se água e dejetos de suínos de forma que o teor de sólidos totais (ST) se mantivesse entre 2,0 e $2,5 \%$.

O substrato utilizado para o abastecimento dos biodigestores foi o mesmo durante todo o experimento, alterando-se apenas o volume do afluente com e sem a separação da fração sólida. $\mathrm{O}$ volume do afluente foi determinado segundo o volume do biodigestor e o tempo de retenção hidráulica adotado (volume do reator/TRH). A separação física da fração sólida do substrato foi feita utilizando-se de peneira com $1 \mathrm{~mm}$ de malha quadrada.

Os biodigestores tubulares semicontínuos foram constituídos de duas partes distintas, sendo um deles o recipiente com o material em fermentação e o outro o gasômetro. O recipiente com o material em fermentação foi composto por um cilindro de PVC, com diâmetro de $30 \mathrm{~cm}$ e comprimento igual a $100 \mathrm{~cm}$, tendo as extremidades fixadas em duas placas de PVC, com 1,5 cm de espessura em cada lado. Em uma das placas, foi fixado apenas um cano para a entrada do material (onde se fez o abastecimento) e na outra placa dois canos, sendo um destinado à saída do biofertilizante e outro à saída do gás.

O gasômetro foi constituído por dois cilindros de 25 e $30 \mathrm{~cm}$ de diâmetro, que se encontravam inseridos um dentro do outro, de tal forma que o espaço existente entre a parede externa do cilindro interior e a parede interna do cilindro exterior comportava um volume de água ("selo de água"), sendo que ambos possuíam o comprimento de $50 \mathrm{~cm}$. O cilindro de $30 \mathrm{~cm}$ de diâmetro foi fixado sobre uma placa de PVC com 2,5 cm de espessura, recebendo o cilindro de $25 \mathrm{~cm}$ de diâmetro no seu interior. O cilindro de $25 \mathrm{~cm}$ de diâmetro teve uma das extremidades vedadas com uma tampa que recebia o gás produzido, sendo a outra extremidade emborcada no selo de água para armazenar o gás produzido. Os gasômetros foram dispostos sobre uma bancada, em condições de temperatura ambiente, abrigados da luz solar e chuvas.

Os teores de sólidos totais (ST) e sólidos voláteis (SV) das amostras coletadas durante os ensaios de caracterização e biodigestão anaeróbia foram determinados de acordo com metodologia descrita por APHA (2005).

Os números mais prováveis de coliformes totais e fecais foram avaliados no afluente e no efluente dos biodigestores semicontínuos, por meio da técnica de tubos múltiplos, a partir de metodologia descrita pela APHA (2005).

Os valores da DQO foram obtidos por método colorimétrico, empregando-se espectrofotômetro, modelo DR/2000 da HACH, e bloco digestor para DQO, também da HACH. A metodologia descrita nos manuais do aparelho faz uso de digestão ácida em meio com dicromato de potássio e catalisadores, utilizando-se da reta-padrão existente na memória do aparelho. Essa metodologia equivale-se à descrita no APHA (2005).

Os valores da DBO foram calculados pela diferença do oxigênio inicial da amostra (mensurado imediatamente após a colheita) pelo oxigênio final (calculado após incubação da amostra por 5 dias em estufa de $\mathrm{BDO}$ a $20^{\circ} \mathrm{C}$ ) e dividido pelo percentual de diluição. Os valores de oxigênio foram calculados com base na metodologia de WINKLER. Essa metodologia equivale-se à descrita no APHA (2005).

Para a determinação do volume de biogás produzido diariamente, mediu-se o deslocamento vertical do gasômetro, que tinha área da seção transversal interna de $0,0507 \mathrm{~m}^{2}$. Após cada leitura, os gasômetros foram zerados, utilizando-se do registro de descarga do biogás. A correção do volume de biogás para as condições de $1 \mathrm{~atm}$ e $20^{\circ} \mathrm{C}$ foi efetuada com base no trabalho de CAETANO (1985), no qual, pelo fator de compressibilidade (Z), o biogás apresenta comportamento próximo ao ideal.

Conforme descrito por SANTOS (1997), para a correção do volume de biogás, utilizou-se da expressão resultante da combinação das leis de Boyle e Gay-Lussac [eq.(1)]: 


$$
\frac{\mathrm{V}_{\mathrm{o}} \mathrm{P}_{\mathrm{o}}}{\mathrm{T}_{\mathrm{o}}}=\frac{\mathrm{V}_{1} \mathrm{P}_{1}}{\mathrm{~T}_{1}}
$$

em que,

$\mathrm{V}_{\mathrm{o}}$ - volume de biogás corrigido, $\mathrm{m}^{3}$;

$\mathrm{P}_{\mathrm{o}}$ - pressão corrigida do biogás, $10322,72 \mathrm{~mm}$ de $\mathrm{H}_{2} \mathrm{O}$;

$\mathrm{T}_{\mathrm{o}^{-}}$temperatura corrigida do biogás, $293,15^{\circ} \mathrm{K}$;

$\mathrm{V}_{1}$ - volume do gás no gasômetro;

$\mathrm{P}_{1}$ - pressão do biogás no instante da leitura, $9652,10 \mathrm{~mm}$ de $\mathrm{H}_{2} \mathrm{O}$, e

$\mathrm{T}_{1}$ - temperatura do biogás, em ${ }^{\circ} \mathrm{K}$, no instante da leitura.

Considerando-se a pressão atmosférica de Jaboticabal igual a $9.641,77 \mathrm{~mm}$ de $\mathrm{H}_{2} \mathrm{O}$ e pressão conferida pelos gasômetros de 10,33 $\mathrm{mm}$ de $\mathrm{H}_{2} \mathrm{O}$, obtém-se, como resultado, a seguinte expressão para a correção do volume de biogás [eq.(2)]:

$$
\mathrm{Vo}=\frac{\mathrm{V}_{1}}{\mathrm{~T}_{1}} 273,84575
$$

Em cada leitura, mediu-se a temperatura $\left({ }^{\circ} \mathrm{C}\right)$ do biogás por meio da utilização de termômetro digital, que dispunha de uma haste longa de metal para ser introduzida no gasômetro.

Os potenciais de produção de biogás foram calculados utilizando-se dos dados de produção diária e das quantidades de substrato, de ST e SV adicionados nos biodigestores, além das quantidades de SV reduzidas durante o processo de biodigestão anaeróbia. Os valores foram expressos em $\mathrm{m}^{3} \mathrm{de}$ biogás por kg de substrato de dejetos ou de ST e SV.

A composição do biogás produzido no biodigestor abastecido com água residuária de suinocultura foi realizada semanalmente para a determinação dos teores de metano $\left(\mathrm{CH}_{4}\right)$ e de dióxido de carbono $\left(\mathrm{CO}_{2}\right)$ em cromatógrafo de fase gasosa Finigan GC-2001, equipado com as colunas Porapack Q, peneira molecular e detector de condutividade térmica.

As determinações dos conteúdos de fibras em detergentes neutro e ácido, celulose e lignina foram efetuadas conforme metodologias propostas por SILVA \& QUEIROZ (2006).

As amostras coletadas durante o desenvolvimento do experimento de biodigestão foram pré-secadas a $60^{\circ} \mathrm{C}$, em estufa de circulação forçada de ar, por $48 \mathrm{~h}$. Depois de secas, foram moídas em moinho de facas e digeridas com ácido sulfúrico $\left(\mathrm{H}_{2} \mathrm{SO}_{4}\right)$ e peróxido de hidrogênio $\left(\mathrm{H}_{2} \mathrm{O}_{2}\right.$ a $\left.50 \%\right)$. Na amostra digerida, foram determinados teores de nitrogênio, fósforo, potássio, cálcio, magnésio, sódio, ferro, cobre, zinco e manganês, segundo APHA (2005). Os teores de fósforo foram determinados pelo método colorimétrico, utilizando-se de espectrofotômetro HACH, modelo DR-2000. O método baseiase na formação de composto amarelo do sistema vanadomolibdofosfórico em acidez de 0,2 a 1,6 N, em que a cor desenvolvida é medida em espectrofotômetro, determinando-se, assim, a concentração de fósforo das amostras, por meio da utilização de uma reta-padrão traçada previamente a partir de concentrações conhecidas, com padrões de concentrações entre 0 e $52 \mathrm{mg}$ de $\mathrm{P} \mathrm{mL}^{-1}$. As concentrações de $\mathrm{K}, \mathrm{Ca}, \mathrm{Mg}, \mathrm{Na}, \mathrm{Fe}, \mathrm{Cu}, \mathrm{Zn}$ e Mn foram determinadas em espectrofotômetro de absorção atômica.

Apesar de o experimento ter sido conduzido por 12 semanas, a análise estatística foi empregada com os dados coletados após a oitava semana (período em que todos os biodigestores atingiram a estabilidade), a fim de obter maior confiabilidade dos resultados.

O delineamento utilizado foi o inteiramente casualizado, com medidas repetidas no tempo, tendo o tempo como subparcelas na análise do experimento, considerando-se os dois tipos de substrato (efluente de biodigestores semicontínuos SSFS e CSFS) em quatro tempos de retenção hidráulica (15; 22; 29 e 36 dias), e as quatro últimas semanas de medição. As médias dos tratamentos foram comparadas entre si pelo teste de Tukey, a 1\% de significância. A análise estatística foi feita no programa SAS (2001). 


\section{RESULTADOS E DISCUSSÃO}

De acordo com os resultados apresentados na Tabela 1, não foram observadas reduções distintas $(\mathrm{P}>0,01)$ de ST e SV entre os biodigestores abastecidos com os afluentes SSFS e CSFS. O mesmo comportamento foi observado por AMARAL et al. (2004), ao estudarem as reduções de ST e SV durante a biodigestão anaeróbia dos dejetos de bovinos nos TRHs de 20; 30 e 40 dias. Já para os valores de DQO e DBO, foram observadas reduções significativas $(\mathrm{P}<0,01)$ entre os biodigestores abastecidos com SSFS e CSFS e também sobre os TRHs, em que o afluente SSFS com o TRH de 36 dias apresentou maiores reduções de DQO e DBO, sendo os valores iguais a 81,70 e $68,42 \%$, respectivamente.

TABELA 1. Redução de ST, SV, DQO e DBO nos biodigestores submetidos a TRH de 15; 22; 29 e 36 dias, com e sem separação da fração sólida. Reduction of TS, VS, COD and BOD in biodigestors undergoing HRT 15; 22; 29 and 36 days, with and without separation of the solid fraction.

\begin{tabular}{|c|c|c|c|c|c|c|c|c|}
\hline \multirow{3}{*}{ TRH } & \multicolumn{8}{|c|}{ Redução \% } \\
\hline & \multicolumn{2}{|c|}{ ST } & \multicolumn{2}{|c|}{ SV } & \multicolumn{2}{|c|}{$\mathrm{DQO}$} & \multicolumn{2}{|c|}{$\mathrm{DBO}$} \\
\hline & SSFS & CSFS & SSFS & CSFS & SSFS & CSFS & SSFS & CSFS \\
\hline 15 & 55,55 & 53,89 & 64,04 & 59,51 & $61,69 \mathrm{Ac}$ & $61,77 \mathrm{Ab}$ & $41,22 \mathrm{Ac}$ & $51,21 \mathrm{Bb}$ \\
\hline 22 & 71,71 & 59,43 & 76,29 & 65,74 & $72,74 \mathrm{Ab}$ & $61,75 \mathrm{Bb}$ & $58,14 \mathrm{Ab}$ & $51,21 \mathrm{Bb}$ \\
\hline 29 & 66,77 & 64,68 & 70,82 & 72,02 & $78,32 \mathrm{Aa}$ & $65,68 \mathrm{Bab}$ & $66,72 \mathrm{Aa}$ & $59,51 \mathrm{Bab}$ \\
\hline 36 & 66,26 & 65,12 & 70,34 & 72,01 & $81,70 \mathrm{Aa}$ & $69,83 \mathrm{Ba}$ & $68,42 \mathrm{Aa}$ & $61,99 \mathrm{Ba}$ \\
\hline C.V.\% & \multicolumn{2}{|c|}{10,03} & \multicolumn{2}{|c|}{7,64} & \multicolumn{2}{|c|}{3,43} & \multicolumn{2}{|c|}{4,23} \\
\hline \multicolumn{9}{|c|}{ Análise de Variância } \\
\hline Afl. $^{\top}$ & \multicolumn{2}{|c|}{$\mathrm{Ns}^{3}$} & \multicolumn{2}{|c|}{ Ns } & \multicolumn{2}{|c|}{$\mathrm{S}^{4}$} & \multicolumn{2}{|c|}{$\mathrm{S}$} \\
\hline TRH & \multicolumn{2}{|c|}{ Ns } & \multicolumn{2}{|c|}{ Ns } & \multicolumn{2}{|c|}{$\mathrm{S}$} & \multicolumn{2}{|c|}{$\mathrm{S}$} \\
\hline Int. $^{2}$ & \multicolumn{2}{|c|}{ Ns } & \multicolumn{2}{|c|}{ Ns } & \multicolumn{2}{|c|}{$\mathrm{S}$} & \multicolumn{2}{|c|}{$\mathrm{S}$} \\
\hline
\end{tabular}

${ }^{1}$ Afluente SSFS ou CSFS; ${ }^{2}$ Interação; ${ }^{3}$ não significativo; ${ }^{4}$ significativo. Na linha, letras maiúsculas comparam resultados com e sem separação da fração sólida e, na coluna, letras minúsculas comparam os tempos de retenção hidráulica. Médias seguidas de letras distintas diferem entre si, pelo teste de Tukey $(\mathrm{P}<0,01)$.

Os valores de produção de biogás (Tabela 2) para o material SSFS foram superiores $(\mathrm{P}<0,01)$ apenas no TRH de 15 dias; para os demais TRHs o material CSFS apresentou os maiores resultados de produção de biogás. AMARAL et al. (2004) atribuíram essas maiores produções totais de biogás, considerando-se TRHs menores, às maiores cargas orgânicas a que esses sistemas são submetidos. Já para os valores de produção de $\mathrm{CH}_{4}$, o material sem sólidos apresentou as maiores produções, independentemente do TRH utilizado. Esse fato pode ser atribuído à maior $(\mathrm{P}<0,01)$ concentração de metano no biogás dos biodigestores abastecidos com o substrato CSFS, fazendo com que sua produção superasse os biodigestores SSFS.

Os potenciais de produção de biogás e de metano (Tabela 3) por kg de ST e SV adicionados foram superiores $(\mathrm{P}<0,01)$ no material CSFS conforme ocorreu acréscimo do TRH. Para o material SSFS, houve aumento de $28 \%$ na produção de biogás $\mathrm{kg}^{-1}$ de ST adicionados quando o TRH passou de 15 para 36 dias. SOUZA \& CAMPOS (2007) também observaram aumento na produção de biogás $\mathrm{kg}^{-1}$ de estrume de suínos de 94,3\% quando o TRH foi alterado de 10 para 30 dias. O melhor desempenho do material CSFS pode ser atribuído à maior quantidade de nutrientes solúveis e ao menor tamanho de partícula (de acordo com os valores apresentados na Tabela 4), ou seja, mais facilmente degradáveis e convertidos em biogás pelos microrganismos no interior dos biodigestores. 
TABELA 2. Produção de biogás, metano e percentagem de metano no dejeto de suíno, com e sem separação da fração sólida, nos TRH de 15, 22, 29 e 36 dias. Production of biogas, methane and methane in the percentage of pig waste with and without separation of the solid fraction in HRT 15; 22; 29 and 36 days.

\begin{tabular}{ccccccc}
\hline \multirow{2}{*}{ TRH } & \multicolumn{2}{c}{$\mathrm{m}^{3}$ Biogás Semana ${ }^{-1}$} & \multicolumn{2}{c}{$\mathrm{m}^{3}$ Metano Semana } & \multicolumn{2}{c}{$\%$ Metano } \\
& SSFS & CSFS & SSFS & CSFS & SSFS & CSFS \\
\hline 15 & $0,32 \mathrm{Aa}$ & $0,30 \mathrm{Ba}$ & $0,20 \mathrm{Aa}$ & $0,21 \mathrm{Aa}$ & $63,50 \mathrm{Bd}$ & $71,62 \mathrm{Ac}$ \\
22 & $0,24 \mathrm{Bb}$ & $0,27 \mathrm{Ab}$ & $0,15 \mathrm{Bb}$ & $0,19 \mathrm{Ab}$ & $64,60 \mathrm{Bc}$ & $73,53 \mathrm{Ab}$ \\
29 & $0,19 \mathrm{Bc}$ & $0,22 \mathrm{Ac}$ & $0,13 \mathrm{Bc}$ & $0,16 \mathrm{Ac}$ & $66,55 \mathrm{Bb}$ & $73,42 \mathrm{Ab}$ \\
36 & $0,17 \mathrm{Ad}$ & $0,18 \mathrm{Ad}$ & $0,12 \mathrm{Bc}$ & $0,14 \mathrm{Ad}$ & $68,58 \mathrm{Ba}$ & $75,36 \mathrm{Aa}$ \\
\hline C.V.\% & \multicolumn{3}{c}{3,18} & \multicolumn{2}{c}{3,92} & \multicolumn{2}{c}{2,28} \\
\hline
\end{tabular}

Na linha, letras maiúsculas comparam resultados com e sem separação da fração sólida, e na coluna, letras minúsculas comparam os tempos de retenção hidráulica. Médias seguidas de letras distintas diferem entre si, pelo teste de Tukey $(\mathrm{P}<0,01)$.

TABELA 3. Potenciais de produção de biogás e de metano com relação aos sólidos totais e voláteis adicionados no dejeto de suíno, com e sem separação da fração sólida, nos TRHs de $15 ; 22 ; 29$ e 36 dias. Potential production of biogas and methane regarding the total and volatile solids added in pig waste with and without separation of the solid fraction in HRT 15; $22 ; 29$ and 36 days.

\begin{tabular}{ccccccccc}
\hline \multirow{2}{*}{ TRH } & \multicolumn{2}{c}{$\mathrm{m}^{3}$ Biogás kg } & -1 & \multicolumn{2}{c}{$\mathrm{m}^{3}$ Metano $\mathrm{kg}^{-1} \mathrm{ST}$} & \multicolumn{2}{c}{$\mathrm{m}^{3}$ Biogás $\mathrm{kg}^{-1} \mathrm{SV}$} & \multicolumn{2}{c}{$\mathrm{m}^{3}$ Metano $\mathrm{kg}^{-1} \mathrm{SV}$} \\
& SSFS & CSFS & SSFS & CSFS & SSFS & CSFS & SSFS & CSFS \\
\hline 15 & $0,716 \mathrm{Bc}$ & $1,394 \mathrm{Aa}$ & $0,455 \mathrm{Bc}$ & $0,998 \mathrm{Ab}$ & $0,664 \mathrm{Bc}$ & $0,890 \mathrm{Ad}$ & $0,469 \mathrm{Bc}$ & $0,746 \mathrm{Ad}$ \\
22 & $0,747 \mathrm{Bc}$ & $1,252 \mathrm{Ab}$ & $0,483 \mathrm{Bc}$ & $0,921 \mathrm{Ac}$ & $0,693 \mathrm{Bc}$ & $1,118 \mathrm{Ac}$ & $0,498 \mathrm{Bc}$ & $0,961 \mathrm{Ac}$ \\
29 & $0,814 \mathrm{Bb}$ & $1,395 \mathrm{Aa}$ & $0,542 \mathrm{Bb}$ & $1,024 \mathrm{Ab}$ & $0,757 \mathrm{Bb}$ & $1,243 \mathrm{Ab}$ & $0,561 \mathrm{Bb}$ & $1,068 \mathrm{Ab}$ \\
36 & $0,911 \mathrm{Ba}$ & $1,443 \mathrm{Aa}$ & $0,625 \mathrm{Ba}$ & $1,087 \mathrm{Aa}$ & $0,846 \mathrm{Ba}$ & $1,287 \mathrm{Aa}$ & $0,646 \mathrm{Ba}$ & $1,134 \mathrm{Aa}$ \\
\hline C.V.\% & \multicolumn{2}{c}{12,65} & \multicolumn{2}{c}{12,67} & \multicolumn{2}{c}{3,21} & \multicolumn{2}{c}{3,01} \\
\hline
\end{tabular}

Na linha, letras maiúsculas comparam resultados com e sem separação da fração sólida, e na coluna, letras minúsculas comparam os tempos de retenção hidráulica. Médias seguidas de letras distintas diferem entre si, pelo teste de Tukey $(\mathrm{P}<0,01)$.

Dessa forma, o processo de separação da fração sólida poderia baratear o custo de implantação de sistemas de biodigestão e ainda aperfeiçoar a operação em projetos subdimensionados ou que se tornaram subdimensionados devido ao incremento na produção de suínos. Entretanto, essa alternativa deve ser estudada com cautela, pois a fração sólida retida na peneira ainda possui potencial poluidor considerável e, dessa forma, necessita de atenção, tanto quanto a fração líquida.

$\mathrm{Na}$ Tabela 4, estão apresentados os teores de fibra em detergente neutro (FDN), fibra em detergente ácido (FDA), lignina, celulose e cinza (em \% dos ST) do material sem separação da fração sólida, com separação da fração sólida e do retido na peneira. Por meio desses resultados, observa-se a eficiência do peneiramento na separação da fração fibrosa da água residuária de suinocultura.

RICO et al. (2006) observaram porcentagens de 7,3 e 5,7; 18,5 e 14,8; 6,0 e 24,2; 4,7 e 20,9 para gordura, proteína, celulose, hemicelulose na fração líquida (separada com peneira de 1,3 mm de malha) e no dejeto in natura de bovinos, respectivamente. No mesmo trabalho, os autores observaram potenciais de produção de biogás de 0,604 e 0,3711 litros $\mathrm{CH}_{4} \mathrm{~g}^{-1} \mathrm{SV}$ para as frações liquida e sólida, respectivamente. 
TABELA 4. Teores de fibra em detergente neutro (FDN), fibra em detergente ácido (FDA), lignina, celulose e cinza em percentagem dos sólidos totais (ST) do material sem separação da fração sólida, com separação da fração sólida e do retido na peneira. Levels of neutral detergent fiber (NDF), acid detergent fiber (ADF), lignin, cellulose and ash as \% of total solid (TS) of the material without separation of the solid fraction, with separation of the solid fraction and the withholding sieve.

\begin{tabular}{crrrrr}
\hline \multirow{2}{*}{ Amostra } & FDN & FDA & Lignina & Celulose & \multicolumn{1}{c}{ Cinza } \\
\cline { 2 - 6 } & \multicolumn{5}{c}{$\%$ ST } \\
SSFS & 31,69 & 13,50 & 3,61 & 9,89 & 12,10 \\
CSFS & 20,27 & 8,65 & 1,67 & 6,98 & 16,19 \\
Retido na peneira & 81,17 & 19,69 & 1,84 & 17,85 & 16,46 \\
\hline
\end{tabular}

MOLLER et al. (2004) estudaram as formas de separação das frações sólidas dos efluentes de suinocultura e encontraram potenciais de produção de 210 litros de $\mathrm{CH}_{4} \mathrm{~kg}^{-1}$ de $\mathrm{SV}$ para a fração sólida dos dejetos, resultante da centrifugação, 247 litros $\mathrm{CH}_{4} \mathrm{~kg}^{-1}$ de $\mathrm{SV}$ para fração sólida dos dejetos, resultante da precipitação química, e 506 litros $\mathrm{CH}_{4} \mathrm{~kg}^{-1}$ de $\mathrm{SV}$ para fração líquida dos dejetos, resultante da centrifugação.

Em todos os tratamentos avaliados, foram observadas reduções no NMP de coliformes totais e fecais (Tabela 5), entretanto não foram verificadas diferenças significativas $(\mathrm{P}>0,01)$ entre os diversos tratamentos. Contudo, o conteúdo de coliformes no biofertilizante restringe sua utilização para algumas finalidades, de acordo com resolução CONAMA 357 (2005), que admite como concentração permissível para a irrigação de hortaliças, que são consumidas cruas, e de frutas que se desenvolvam rentes ao solo e que sejam ingeridas cruas sem remoção de película, quantidades inferiores a 200 coliformes $100 \mathrm{~mL}^{-1}$ de biofertilizante. As reduções encontradas neste trabalho foram superiores às verificadas por AMORIM et al. (2004), que observaram reduções de apenas 99\% ao estudarem a eliminação de coliformes fecais de resíduos de suínos por meio da digestão anaeróbia mesofílica, em biodigestores contínuos, modelo indiano, com TRH de 18 dias. Essa maior redução pode estar relacionada ao modelo de biodigestor utilizado, visto que o biodigestor tubular se caracteriza por apresentar elevada produção de ácido na entrada do biodigestor, sendo que a ação do $\mathrm{pH}$ se dá logo no início do processo, reduzindo, assim, a população de coliformes no material de entrada.

TABELA 5. Número mais provável (NMP $100 \mathrm{~mL}^{-1}$ ) de coliformes totais (CT) e fecais (CF) durante a biodigestão anaeróbia, com dejeto SSFS e CSFS, nos TRHs de 15; 22; 29 e 36 dias. Most probable number (NMP $100 \mathrm{~mL}^{-1}$ ) of total coliform (TC) and fecal (FC) during the anaerobic digestion with waste and SSFS CSFS in HRT 15; 22; 29 and 36 days.

\begin{tabular}{|c|c|c|c|c|c|c|}
\hline \multirow{3}{*}{ TRH } & \multicolumn{6}{|c|}{$\mathrm{CT}$} \\
\hline & \multicolumn{3}{|c|}{ SSFS } & \multicolumn{3}{|c|}{ CSFS } \\
\hline & Afluente & Efluente & Redução (\%) & Afluente & Efluente & Redução (\%) \\
\hline 15 & $9,110^{9}$ & $1,510^{4}$ & 99,99984 & $3,610^{9}$ & $4,610^{4}$ & 99,99872 \\
\hline 22 & $9,110^{9}$ & $1,110^{5}$ & 99,99879 & $3,610^{9}$ & $4,610^{4}$ & 99,99872 \\
\hline 29 & $9,110^{9}$ & $1,110^{5}$ & 99,99879 & $3,610^{9}$ & $4,310^{3}$ & 99,99988 \\
\hline 36 & $9,110^{9}$ & $4,610^{4}$ & 99,99949 & $3,610^{9}$ & $3,610^{2}$ & 99,99999 \\
\hline \multirow{3}{*}{ TRH } & \multicolumn{6}{|c|}{$\mathrm{CF}$} \\
\hline & \multicolumn{3}{|c|}{ SSFS } & \multicolumn{3}{|c|}{ CSFS } \\
\hline & Afluente & Efluente & Redução (\%) & Afluente & Efluente & Redução (\%) \\
\hline 15 & $7,310^{9}$ & $1,510^{4}$ & 99,99979 & $3,610^{9}$ & $4,610^{4}$ & 99,99872 \\
\hline 22 & $7,310^{9}$ & $1,110^{5}$ & 99,99849 & $3,610^{9}$ & $1,510^{4}$ & 99,99958 \\
\hline 29 & $7,310^{9}$ & $4,610^{4}$ & 99,99936 & $3,610^{9}$ & $9,110^{2}$ & 99,99997 \\
\hline 36 & $7,310^{9}$ & $1,510^{4}$ & 99,99979 & $3,610^{9}$ & $3,610^{2}$ & 99,99999 \\
\hline
\end{tabular}


STEIL (2001) também observou reduções de 99,99\% em substratos preparados a partir dos dejetos de suínos, utilizando $0 ; 10$ e $15 \%$ de inóculo e 154 dias de retenção, em concentração inicial de $8 \%$ de ST.

Observando-se os resultados apresentados na Tabela 6, verifica-se que houve acréscimo na concentração dos macro e micronutrientes no efluente em comparação com o afluente, com exceção dos teores de $\mathrm{N}, \mathrm{Fe}, \mathrm{Zn}$ e $\mathrm{Cu}$ para o substrato CSFS. Esse fato era esperado em função da redução da fração orgânica, por meio da produção de biogás, acarretando, assim, a concentração dos constituintes inorgânicos.

Com base nestes resultados, destaca que houve diferença significativa $(\mathrm{P}<0,01)$ apenas com relação ao tipo de efluente utilizado no abastecimento, em que os afluentes SSFS apresentaram os menores teores de nutrientes no efluente, em comparação com CSFS.

TABELA 6. Valores médios dos teores de N, P, K, Mg, Ca, Na, Zn, Fe, Mn e Cu (em \% dos ST) no afluente e no efluente dos biodigestores abastecidos com dejeto de suíno, com e sem separação da fração sólida. Average values of the levels of $\mathrm{N}, \mathrm{P}, \mathrm{K}, \mathrm{Mg}, \mathrm{Ca}, \mathrm{Na}, \mathrm{Zn}$, $\mathrm{Fe}, \mathrm{Mn}$ and $\mathrm{Cu}$ (in \% of ST) in the affluent and the effluent biodigestors supplied with pig waste, with and without separation of the solid fraction.

\begin{tabular}{|c|c|c|c|c|c|c|c|c|c|c|}
\hline & $\mathrm{N}$ & $\mathrm{P}$ & $\mathrm{K}$ & $\mathrm{Mg}$ & $\mathrm{Ca}$ & $\mathrm{Na}$ & $\mathrm{Zn}$ & $\mathrm{Fe}$ & $\mathrm{Mn}$ & $\mathrm{Cu}$ \\
\hline & \multicolumn{10}{|c|}{$\%$ dos ST } \\
\hline Afluente SSFS & 3,737 & 2,047 & 2,324 & 0,591 & 1,158 & 0,660 & 0,064 & 0,266 & 0,053 & 0,020 \\
\hline Efluente SSFS 15 dias & 3,841 & 4,844 & 7,033 & 1,212 & 1,364 & 1,966 & 0,055 & 0,224 & 0,065 & 0,018 \\
\hline Efluente SSFS 22 dias & 3,858 & 5,100 & 6,776 & 1,307 & 1,290 & 1,840 & 0,056 & 0,233 & 0,070 & 0,019 \\
\hline Efluente SSFS 29 dias & 3,847 & 4,610 & 6,607 & 1,191 & 1,475 & 1,847 & 0,064 & 0,223 & 0,069 & 0,021 \\
\hline SFS 36 dias & 3,812 & 4,589 & 6,529 & 1,168 & 1,449 & 1,740 & 0,062 & 0,219 & 0,069 & 0,022 \\
\hline Afluente CSFS & 4,522 & 2,618 & 2,952 & 0,702 & 1,376 & 0,767 & 0,080 & 0,331 & 0,065 & 0,025 \\
\hline FS 15 dias & 3,716 & 4,067 & 7,351 & 0,948 & 1,703 & 2,141 & 0,062 & 0,251 & 0,080 & 0,026 \\
\hline SFS 22 dias & 3,470 & 3,962 & 7,619 & 0,944 & 1,816 & 2,082 & 0,059 & 0,234 & 0,076 & 0,026 \\
\hline dias & 3,382 & 3,929 & 7,938 & 0,963 & 1,875 & 2,084 & 0,062 & 0,215 & 0,074 & 0,024 \\
\hline Efluente CSFS 36 dias & 3,347 & 3,880 & 7,925 & 0,947 & 1,830 & 2,212 & 0,060 & 0,212 & 0,071 & 0,024 \\
\hline Retido na peneira & 1,546 & 0,501 & 0,390 & 0,300 & 0,571 & 0,125 & 0,020 & 0,169 & 0,019 & 0,007 \\
\hline \multicolumn{11}{|c|}{ Análise de Variância } \\
\hline Afluente & S & S & $\mathrm{S}$ & $\mathrm{S}$ & $\mathrm{S}$ & S & $\mathrm{S}$ & $\mathrm{S}$ & $\mathrm{S}$ & S \\
\hline & NS & NS & NS & NS & NS & NS & NS & NS & NS & NS \\
\hline Interação & NS & NS & NS & NS & NS & NS & NS & NS & NS & NS \\
\hline
\end{tabular}

S: significativo $(\mathrm{P}<0,01)$, NS: não significativo $(\mathrm{P}>0,01)$.

É importante considerar que o aumento da concentração de nutrientes no material CSFS pode ter contribuído para melhorias nas produções de biogás, pois pode ter caracterizado um meio mais propício ao desenvolvimento dos microrganismos anaeróbios, assim como permitido a obtenção de efluente com maior concentração de elementos fertilizantes.

\section{CONCLUSÕES}

A separação da fração sólida da água residuária de suinocultura foi importante para maximizar a eficiência dos biodigestores, proporcionando maior potencial de produção de biogás, maior teor de metano no biogás e biofertilizante com maiores teores de elementos minerais.

\section{REFERÊNCIAS}

AHNA, J.; DOA, T. H.; KIMB, S.D.; HWANG, S. The effect of calcium on the anaerobic digestion treating swine wastewater. Biochemical Engineering Journal, Manchester, v.30, n.1, p.33-38, 2006. 
ALVAREZ, R.; GUNNAR, L. Semi-continuous co-digestion of solid slaughterhouse waste, manure, and fruit and vegetable waste. Renewable Energy, Great Britain, v.33, n.2, p.726-734, 2008.

AMARAL, C.M.C.; AMARAL, L.A.; LUCAS JÚNIOR, J.; NASCIMENTO, A.A.; FERREIRA, D.S.; MACHADO, M.R.F. Biodigestão anaeróbia de dejetos de bovinos leiteiros submetidos a diferentes tempos de retenção hidráulica. Ciência Rural, Santa Maria, v.34, n.6, p.1.897-1.902, 2004.

APHA. AMERICAN PUBLIC HEALTH ASSOCIATION. Standard methods for examination of water and wastewater. $21^{\text {th }}$ ed. Washington: American Water Works Association, 2005. $1.368 \mathrm{p}$.

AMORIM, A.C.; LUCAS JÚNIOR, J.; RESENDE, K.T. Biodigestão anaeróbia de dejetos de caprinos obtidos nas diferentes estações do ano. Engenharia Agrícola, Jaboticabal, v.24, n.1, p.1624, 2004.

\section{ABIPECS. ASSOCIAÇÃO BRASILEIRA DA INDÚSTRIA PRODUTORA E EXPORTADORA} DE CARNE SUÍNA. Disponível em: <http://www.abipecs.org.br/>. Acesso em: 16 dez. 2007.

CAETANO, L. Proposição de um sistema modificado para quantificação de biogás. $1985.75 \mathrm{f}$. Dissertação (Mestrado em Energia na Agricultura) - Faculdade de Ciências Agronômicas, Universidade Estadual Paulista, Botucatu, 1985.

CONAMA. CONSELHO NACIONAL DO MEIO AMBIENTE. Padrões de qualidade para os parâmetros monitorados na rede de monitoramento, segundo Resolução CONAMA 357/2005. Disponível em: http:<//www.mma.gov.br/port/conama/res/res05/>. Acesso em: 11 dez. 2007.

CÔTÉ, C.; MASSÉ, I.D.; QUESSY, S. Reduction of indicator and pathogenic microorganisms by psychrophilic anaerobic digestion in swine slurries. Bioresource Technology, Oxford, v.97, n.3, p.686-691, 2006.

MOLLER, H.B.; SOMMER, S.G.; AHRING, B.K. Methane productivity of manure, straw and solid fractions of manure. Biomass Bioenergy, Boulder, v.26, n.5, p.485-495, 2004.

ORRICO, A.C.A.; LUCAS JÚNIOR, J.; ORRICO JÚNIOR, M.A.P. Caracterização e biodigestão anaeróbia dos dejetos de caprinos. Engenharia Agrícola, Jaboticabal, v.27, n.3, p.639-647, 2007.

RICO, J.L.; GARCIA, H.; RICO, C.; TEJERO, I. Characterisation of solid and liquid fractions of dairy manure with regard to their component distribution and methane production. Bioresource Technology, Oxford, v.98, n.3, p.971-979, 2006.

SANTOS, T.M.B. Caracterização química, microbiológica e potencial de produção de biogás a partir de três tipos de cama, considerando dois ciclos de criação de frangos de corte. $1997.95 \mathrm{f}$. Dissertação (Mestrado em Produção Animal) - Faculdade de Ciências Agrárias e Veterinárias, Universidade Estadual Paulista, Jaboticabal, 1997.

SILVA, D.J.; QUEIROZ, A.C. Análise de alimentos: métodos químicos e biológicos. 3.ed. Viçosa -MG: Editora Universitária, 2006. 166 p.

SOUZA, C.F.; CAMPOS, J.A. Avaliação do tempo de retenção hidráulica, agitação e temperatura em biodigestores operando com dejetos de suínos. Revista Brasileira de Agroecologia, Porto Alegre, v.2, n.1, 2007.

STATISTICAL ANALYSIS SYSTEM - SAS. SAS system for Windows. Versão 8.2. Cary, 2001.

STEIL, L. Avaliação do uso de inóculos na biodigestão anaeróbia de resíduos de aves de postura, frangos de corte e suínos. 2001. 108 f. Dissertação (Mestrado em Biotecnologia) - Instituto de Química, Universidade Estadual Paulista, Araraquara, 2001. 